\title{
647.
}

\section{ON THE QUARTIC SURFACES REPRESENTED BY THE EQUATION, SYMMETRICAL DETERMINANT $=0$.}

[From the Quarterly Journal of Pure and Applied Mathematics, vol. xIv. (1877), pp. $46-52$.]

Consider the equation

$$
\nabla=\left|\begin{array}{llll}
a, & h, & g, & l \\
h, & b, & f, & m \\
g, & f, & c, & n \\
l, & m, & n, & d
\end{array}\right|=0
$$

where for the moment $(a, b, \ldots)$ denote linear functions of the coordinates $(x, y, z, w)$. This is a quartic surface having 10 nodes; viz. if we write $(A, B, \ldots)$ for the first minors of the determinant, then the cubic surfaces $A=0, B=0, \ldots$ have in common 10 points which are nodes of the quartic surface.

Suppose that $(a, b, c, f, g, h)$ are linear functions of the form $(x, y)$; then, observing that every term of $\nabla$ contains as a factor

$$
\left|\begin{array}{lll}
a, & h, & g \\
h, & b, & f \\
g, & f, & c
\end{array}\right|
$$

or one of its first minors, it is clear that the line $x=0, y=0$ is a double line on the surface. But the number of nodes is now less than 10 ; in fact, writing $(x=0$, $y=0$ ), we make each of the first minors of $\nabla$ to vanish; that is, the cubic surfaces, which by their intersection determine the nodes, have in common the line $(x=0, y=0)$, and there is a diminution in the number of their common intersections. I do not pursue the enquiry, but pass to a different question. 
I, in fact, take the terms $(a, \ldots)$ of the determinant to be homogeneous functions of $(x, y, z, w)$ of the degrees

$$
\left|\begin{array}{llll}
0, & 1, & 1, & 0 \\
1, & 2, & 2, & 1 \\
1, & 2, & 2, & 1 \\
0, & 1, & 1, & 0
\end{array}\right|
$$

respectively, viz. $a, d, l$ are constants, $g, h, m, n$ linear functions, and $b, c, f$ quadric functions of the coordinates; $\nabla=0$ still represents a quartic surface; and it appears by a general formula that the number of nodes is $=8$. But we can easily show this directly; and further, that the 8 nodes are the intersections of three quadric surfaces; or say that the quartic surface is octadic. For denoting as before the first minors by $A, \ldots$, then $B, C, F$ are each of them a quadric function of the coordinates, viz. we have

$$
\begin{aligned}
& B=d\left(a c-g^{2}\right)-c l^{2}-a n^{2}+2 g l n \\
& C=d\left(a b-h^{2}\right)-a m^{2}-b l^{2}+2 h l m \\
& F=d(g h-a f)+l^{2} f+m n a-n l h-l m g
\end{aligned}
$$

and we have identically

$$
B C-F^{2}=\left(a d-l^{2}\right) \nabla,
$$

so that throwing out the constant factor $a d-l^{2}$, the equation of the surface is

$$
B C-F^{2}=0
$$

and it has 8 nodes, the intersections of the three quadric surfaces $B=0, C=0, F=0$. By equating to zero any other minor of the determinant $\nabla$, we have a surface passing through the 8 nodes; we have for instance the quartic surface

$$
\left|\begin{array}{lll}
a, & h, & g \\
h, & b, & f \\
g, & f, & c
\end{array}\right|=0 .
$$

Suppose now (and in all that follows) that, the degrees being as already mentioned, we further assume that $b, c, f$ are quadric functions of the form $(x, y)^{2}, g, h$ linear functions of the form $(x, y)$; then since each term of $\nabla$ contains either

$$
\left|\begin{array}{lll}
a, & h, & g \\
h, & b, & f \\
g, & f, & c
\end{array}\right|
$$

or one of its first minors, it is clear that the line $(x=0, y=0)$ is a double line on the surface. But in the present case there is not any diminution in the number of the nodes; in fact, writing $x=0, y=0$, and therefore $b, c, f, g, h$ each $=0$ (but not $a=0$ ), the minors $B, C, F$ none of them vanish; that is, the line $x=0, y=0$ is not a line on any one of the quadric surfaces, and the quadric surfaces intersect as before in an octad of points. 
The equation $\nabla=0$ thus represents a quartic surface having a double line, and also 8 nodes forming an octad.

We may without loss of generality write $d=0$; in fact, the determinant is unaltered if we add to the fourth column $\theta$ times the first column, and then to the fourth line $\theta$ times the first line; the determinant is thus of the original form, but in place of $d$ it has $d+2 \theta l+\theta^{2} a$, which by properly determining $\theta$ can be made $=0$. And then changing the original $l, m, n$, the equation is

Or, writing for shortness,

$$
\nabla=\left|\begin{array}{llll}
a, & h, & g, & l \\
h, & b, & f, & m \\
g, & f, & c, & n \\
l, & m, & n, & 0
\end{array}\right|=0 .
$$

$$
K=\left|\begin{array}{lll}
a, & h, & g \\
h, & b, & f \\
g, & f, & c
\end{array}\right|
$$

and denoting the minors hereof by $(a, b, c, f, g, h)$, then the equation is

$$
\nabla=(\mathrm{a}, \mathrm{b}, \mathrm{c}, \mathrm{f}, \mathrm{g}, \mathrm{h} \gamma \mathrm{l}, m, n)^{2}=0,
$$

where the degree of $K$ is 4 , and the degrees of a, b, c, f, g, h are $4,2,2,2,3,3$ respectively, those of $l, m, n$ being $0,1,1$ respectively.

The nodes are, as before, the intersections of the quadric surfaces $B=0, C=0$, $F=0$, viz. $(d$ being now $=0)$ the values are

$$
\begin{aligned}
-B & =c l^{2}-2 g l n+a n^{2}, \\
-C & =b l^{2}-2 h l m+a m^{2}, \\
F & =f l^{2}-g l m-h l n+a m n .
\end{aligned}
$$

But, according to a previous remark, the nodes lie also on the quartic surface $K=0$; viz. this is a set of four planes intersecting in the line $x=0, y=0$.

Now, in general, any plane through the line $x=0, y=0$ meets the surface in this line twice and in a conic; if the plane is $y=\theta x$, we have

$$
a, b, c, f, g, h=a^{\prime}, b^{\prime} x^{2}, c^{\prime} x^{2}, f^{\prime} x^{2}, g^{\prime} x, h^{\prime} x,
$$

where $a^{\prime}, b^{\prime}, c^{\prime}, f^{\prime}, g^{\prime}, h^{\prime}$ are functions of $\theta$ of the degrees $(0,2,2,2,1,1)$ respectively; and thence also

$$
\mathrm{a}, \mathrm{b}, \mathrm{c}, \mathrm{f}, \mathrm{g}, \mathrm{h}=\mathrm{a}^{\prime} x^{4}, \mathrm{~b}^{\prime} x^{2}, \mathrm{c}^{\prime} x^{2}, \mathrm{f}^{\prime} x^{2}, \mathrm{~g}^{\prime} x^{3}, \mathrm{~h}^{\prime} x^{3}
$$

where $a^{\prime}, b^{\prime}, c^{\prime}, f^{\prime}, g^{\prime}, h^{\prime}$ are functions of $\theta$ of the degrees $4,2,2,2,3,3$ respectively; the equation of the surface thus becomes $\left(\mathrm{a}^{\prime}, \mathrm{b}^{\prime}, \mathrm{c}^{\prime}, \mathrm{f}^{\prime}, \mathrm{g}^{\prime}, \mathrm{h}^{\prime} \gamma l x, m, n\right)^{2}=0$; viz. this is a quadric equation which, combined with the equation $y-\theta x=0$, determines the 
conic in question. But for each of the planes $K=0$, we have $\left(\mathrm{a}^{\prime}, \mathrm{b}^{\prime}, \mathrm{c}^{\prime}, \mathrm{f}^{\prime}, \mathrm{g}^{\prime}, \mathrm{h}^{\prime} \gamma l x, m, n\right)^{2}$ a perfect square, or the conic a two-fold line; we have thus the 8 nodes lying in pairs on four lines, say the four "rays," in the four planes $K=0$ respectively; each of these rays meets the double line $x=0, y=0$ in a point; and we have thus on the double line 4 points, which are in fact pinch-points of the surface (as to this presently). It has just been stated that for the plane passing through the nodal line and a ray, the conic is a two-fold line (the ray twice) containing upon it a pair of nodes; more properly, the conic is the point-pair composed of the two nodes. equation

We can find through the nodes four different plane-pairs; in fact, forming the this is

$$
-B+2 \lambda F-\lambda^{2} C=0
$$

$$
l^{2}\left(c+2 \lambda f+\lambda^{2} b\right)-2 l(g+\lambda h)(n+\lambda m)+a(n+\lambda m)^{2}=0
$$

or, as this may also be written,

$$
[a(n+\lambda m)-l(g+\lambda h)]^{2}+l^{2}\left(b-2 \lambda f+\lambda^{2} c\right)=0,
$$

where $\mathrm{b}, \mathrm{c}, \mathrm{f}$ and therefore also $\mathrm{b}-2 \lambda \mathrm{f}+\lambda^{2} \mathrm{c}$ are of the form $(x, y)^{2}$; say that we have $\mathrm{b}-2 \lambda \mathrm{f}+\lambda^{2} \mathrm{c}=(p, q, r \gamma x, y)^{2}$, where $p, q, r$ are of course quadric functions of $\lambda$; determining $\lambda$ by the quartic equation $p r-q^{2}=0$, we have $b-2 \lambda f+\lambda^{2}$ c a perfect square, $=(\alpha x+\beta y)^{2}$ suppose; and we have thus the plane-pair

$$
[a(n+\lambda m)-l(g+\lambda h)]^{2}-l^{2}(\alpha x+\beta y)^{2}=0
$$

containing the eight nodes; viz. there are four such plane-pairs. The two planes of a plane-pair intersect in a line called an "axis"; that is, we have four axes each meeting the nodal line; and we have thus also through the nodal line and the four axes respectively four planes, which are "pinch-planes" of the quartic surface (as to this presently).

It has just been seen that the equation $B-2 \lambda F+C \lambda^{2}=0$ (where $\lambda$ is arbitrary) is expressible in the form

$$
[a(n+\lambda m)-l(g+\lambda h)]^{2}+l^{2}\left(p, q, r \gamma(x, y)^{2}=0,\right.
$$

viz. this is the equation of a quadric cone having its vertex on the nodal line at the point $x=0, y=0$, an $-l g+\lambda(a m-l h)=0$; this is, in fact, a cone touching the surface, as at once appears by writing the equation of the cone in the form

$$
\frac{1}{C}\left\{B C-F^{2}+(\lambda C-F)^{2}\right\}=0
$$

that is,

$$
\frac{1}{C}\left\{-l^{2} \nabla+\left(\lambda C-F^{\prime}\right)^{2}\right\}=0
$$

we thus see that, taking for vertex any point whatever on the nodal line, there is a circumscribed quadric cone. 
For each of the above-mentioned four values of $\lambda$, the quadric cone breaks up into a plane-pair; each plane of the plane-pair is thus a "trope" or plane touching the surface along a conic; viz. this is the conic passing through the intersection of the plane (or say of an axis) with the nodal line and through four nodes of the surface. We have thus 8 tropes, intersecting in pairs in the four axes (and the intersection of each axis with the nodal line being a pinch-point). Moreover, joining the nodes in pairs, we have four rays, each meeting the nodal line, the plane through it and the nodal line being a pinch-plane; this is illustrated in the figure.

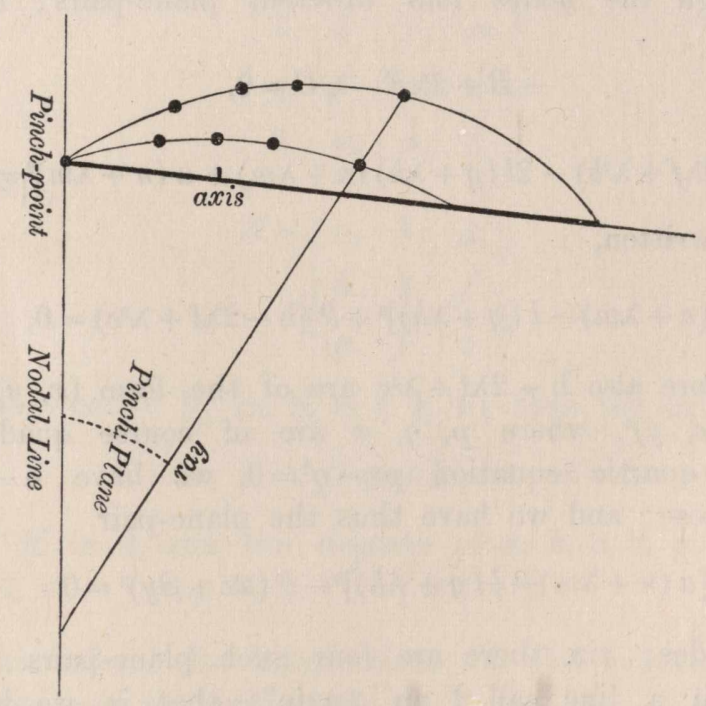

As to the pinch-planes and pinch-points, remark first that a plane through the nodal line is in general a bitangent plane, its two points of contact being the points where the conic in such plane meets the nodal line. When the two points of contact come to coincide, the plane is a pinch-plane; viz. this happens when the plane passes through a ray, the conic being then the ray twice repeated. And secondly, at a point on the nodal line there are in general two tangent planes, viz. these are the tangent planes to the quadric cone belonging to such point; when the two tangent-planes come to coincide the point is a pinch-point, and this happens when the point is the intersection of the nodal line with an axis, for then (the quadric cone breaking up into the two tropes through the axis) the two tangent planes become the plane through the axis taken twice.

Each section through the nodal line is a conic, and the polar of the nodal line in regard to this conic is a point; the locus of this point (for different sections through the nodal line) is a right line which may be called simply the "polar." To prove this, considering the section by the plane $y=\theta x$, we have to find the pole of the line $x=0$ in regard to the conic

$$
\left(\mathrm{a}^{\prime}, \mathrm{b}^{\prime}, \mathrm{c}^{\prime}, \mathrm{f}^{\prime}, \mathrm{g}^{\prime}, \mathrm{h}^{\prime} \gamma l x, m, n\right)^{2}=0 ;
$$


this is $l x: m: n=a^{\prime}: h^{\prime}: g^{\prime}$, viz. if $g=g_{0} x+g_{1} y, h=h_{0} x+h_{1} y$, this is

$$
l x: m: n=a: g_{0}+g_{1} \theta: h_{0}+h_{1} \theta,
$$

or joining hereto the equation $y=\theta x$, we have

$$
l x: l y: m: n=a: a \theta: g_{0}+g_{1} \theta: h_{0}+h_{1} \theta,
$$

where $l, a, g_{0}, g_{1}, h_{0}, h_{1}$ are constants; $m, n$ are linear functions of the coordinates $(x, y, z, w)$. The equations represent, it is clear, a right line which is the polar in question; and they may be written

$$
\frac{l x}{a}=\frac{h_{1} m-g_{1} n}{h_{1} g_{0}-h_{0} g_{1}}, \quad \frac{l y}{a}=-\frac{h_{0} m-g_{0} n}{h_{1} g_{0}-h_{0} g_{1}} .
$$

When the plane passes through a ray, the conic becomes, as was stated, the pointpair composed of the two nodes in such ray; the harmonic in regard to these two points of the intersection of the ray with the nodal line is thus a point on the polar: that is, the polar meets the ray; and the two nodes are situate harmonically in regard to the intersections of the ray with the nodal line and the polar respectively.

The polar may be arrived at in a different manner, viz. if instead of a plane through the nodal line we consider a point on the nodal line, this is the vertex of a circumscribed quadric cone; and taking the polar plane of the nodal line in regard to this cone, then considering the point as variable, the different polar planes all pass through a line which is the polar in question. And hence, taking for the point the intersection of the nodal line with an axis, it appears that the axis meets the polar; and, moreover, that the two tropes through the axis are harmonics in regard to the planes through the axis, and the polar and nodal line respectively.

Collecting the foregoing results, we have a quartic surface as follows:

We have two lines, a nodal line and a polar; meeting each of these, four lines called "rays" and four other lines called "axes." On each ray, harmonically in regard to its intersections with the nodal line and the polar, two nodes of the surface (in all 8 nodes): through each axis, harmonically in regard to the planes through it and the nodal line and the axis respectively, two tropes of the surface (in all 8 tropes). In each trope (or, what is the same thing, in its conic of contact) are 4 nodes; through each node (or, what is the same thing, touching its tangential quadricone) are 4 tropes; the relation of the nodes and tropes may be thus represented, viz. taking the pairs of nodes to be 1,$2 ; 3,4 ; 5,6 ; 7,8$; and those of tropes to be I, II; III, IV; V, VI; VII, VIII ; then we have 


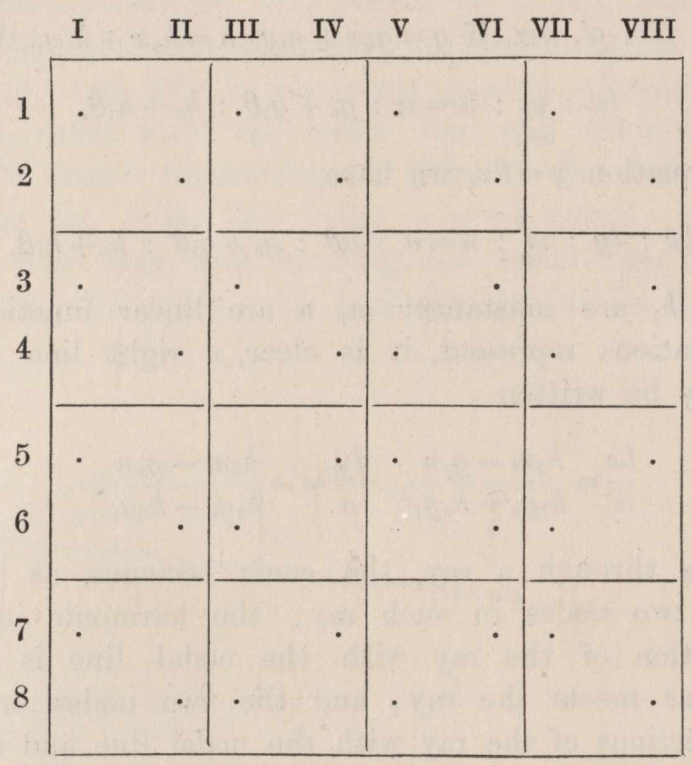

viz. reading horizontally or vertically, the dots show the tropes through each node, or the nodes in each trope.

The plane through any ray and the nodal line is a pinch-plane of the surface, its point of contact being the intersection of the ray with the nodal line; and the intersection of each axis with the nodal line is a pinch-point of the surface, the tangent plane being the plane through the axis and the nodal line; the surface has thus 4 pinch-planes and 4 pinch-points. 